\section{P3.112 USE OF SOCIAL APPS AMONG YOUNG MEN WHO HAVE SEX WITH MEN (YMSM) BETWEEN THE AGES 18 TO 24 AT THE WESTERN REGION OF PUERTO RICO: EVALUATION OF PROTECTIVE AND RISK FACTORS}

Jorge L Rodriguez Lebron, Edna Acosta Pérez, Vivian Colón López. University of Puerto RicoMedical Science Campus, San Juan - Puerto Rico

10.1136/sextrans-2017-053264.347

Introduction Increasing new cases of HIV among Men who have Sex with Men (MSM) has been observed in Puerto Rico (PR). According to the HIV Surveillance System, 23\% of the HIV/AIDS cases are among MSM. Adoption and proliferation of digital platforms have become a new venue for MSM to meet new sexual partners. Researchers have studied these new venues without getting any consensus if the use of social apps are a protective or risk factor for HIV infection. This study aims to understand the use of social apps among young MSM (18-24 years), practices with those who they met online and perceived risk of infection.

Methods Data collected from the needs assessment of the Youth Prevention Program between March and September 2016, was used. A convenience sampling of 183 MSM were recruited in the Western region of PR. Descriptive analysis was used to portray selected characteristics and use of social apps.

Results More than half $(80 \%)$ of the participants reported the use of any social apps for meeting sexual partners in their lifetime. Among those who use apps, 65\% reported was for hook-up. The average number of lifetime sexual partners met by these apps were $5.7 \pm 4.7$ partners. Inconsistent condom use was reported among 40\%, which indicated have not used condom while having sex with partners they met online. The most common app used was Grindr (66\%), followed by Facebook (55\%). When asked their perceived risk of HIV, only $16 \%$ reported they considered to be at high risk of contracting with HIV when meet their sexual partners online. Of further note, $24 \%$ sent photos of their bare buttock, $31 \%$ of their penis and $48 \%$ display their chests.

Conclusion The study intends in the future to demonstrate if there is any association from using these social apps and HIV infection. This preliminary analysis showed that apps are becoming a frequent to meet sexual partners but they don't consider these apps as risk venues for contracting HIV. This study will help strengthen prevention programs and prevention initiatives for high risk populations in the island.

\section{P3.113 HIV-1 DRUG RESISTANCE MUTATIONS IN INFECTED CHILDREN AND ADOLESCENTS FAILING THERAPY: IMPACT IN THE SUSCEPTIBILITY OF DRUGS USED IN SALVAGE THERAPIES}

${ }^{1}$ José Carlos Couto Fernandez, ${ }^{2}$ Carlos Silva De Jesus, ${ }^{2}$ José Henrique Pilotto, ${ }^{2}$ Mariza Gonçalves Morgado. 'Laboratory of AIDS and Molecular Immunology, Oswaldo Cruz Foundation-loc/Fiocruz, Rio de Janeiro - RJ, Brazil; ${ }^{2}$ Oswaldo Cruz Foundation-loc/Fiocruz, Rio de Janeiro - RJ, Brazil

\subsection{6/sextrans-2017-053264.348}

Introduction Paediatric HIV-1 infection remains an important public health issue in resource-limited settings. In Brazil, the access to combined antiretroviral therapy (cART) and the HIV-1 genotyping test are available for all infected children and adolescents. However, mainly due to low patient adherence, multidrug-resistant (MDR) viruses have been increasing over the last years. This study estimate the resistance associated to the new generation protease inhibitors (PIs) and non-nucleoside reverse transcriptase inhibitors (NNRTIs) and the possible use in rescue strategies for children and adolescents failing cART.

Methods Between 2008 and 2014, blood samples from 246 HIV-1-infected children and adolescents failing different cART regimens, were collected in the Rio de Janeiro State, Brazil. The profiles of HIV-1 resistance mutations were evaluated in the Stanford website and subtype confirmed by phylogeny.

Results The majority of genotyped samples were classified as HIV-1 subtype B (75.6\%), followed by subtype F1 (15.4\%), $\mathrm{BF}$ recombinants (4.1\%), subtype $\mathrm{C}(3.3 \%)$ and subtype $\mathrm{A} 1$, CRF 02AG and the recombinant A1B in one subject each. A total of $31.2 \%$ of patients showed resistance associated to first line therapy, $45.3 \%$ for the second line and $23.4 \%$ to third line. MDR mutations were detected in only $3 \%$ of the children. The prevalence of PI-associated mutations was low (3.6\%), except for the M46I/L mutation (24.4\%) associated to the majority of PIs. The resistance to PIs used in the rescue therapies, were $2.8 \%$ for the darunavir and $3.6 \%$ for the tipranavir. High prevalence of thimidine associated mutations (TAMs) and to lamivudine, were observed (>80\%). But, mutations to the nucleotide reverse transcritptase inhibitor (NRTI) Tenofovir, showed low prevalence $(5.3 \%)$. In addition, resistance mutations associated to the decrease of a virological response to etravirine were $5.4 \%$ and $3.8 \%$ to rilpivirine.

Conclusion Low prevalence of drug resistance mutations associated to the new generation of PIs and NNRTIs was observed in our genotyping database. The impact of resistance mutations under darunavir seems lower than for tipranavir in children failing other PI-based regimens. Although prior failure to other PIs or NNRTI might produce cross-resistance, the results show that all of these drugs used in the therapy rescue, could be effective and constitute a good option for children who failure other regimens.

\section{P3.114 ANALYSIS OF NOTIFICATION'S CASES OF CONGENITAL SYPHILIS IN AN UNIVERSITY HOSPITAL FROM NITEROI 2008-2015}

${ }^{1}$ EPN Junior, ${ }^{2}$ LFM Souza, ${ }^{2} \mathrm{PM}$ Monteiro, ${ }^{2} \mathrm{CB}$ Fernandes, ${ }^{2} \mathrm{AS}$ Mota, ${ }^{3} \mathrm{MRL}$ Passos. ${ }^{1}$ Faculdade de Medicina de Campos, Campos - RJ, Brazil; ${ }^{2}$ Faculdade de Medicina da Universidade Federal Fluminense, Niteroi - RJ, Brazil, ${ }^{3}$ Setor de DST da Universidade Federal Fluminense, Niteroi - RJ, Brazil

\subsection{6/sextrans-2017-053264.349}

Introduction Congenital syphilis (CS) is a serious public health problem in Brazil, being the cause of fetal death and other perinatal complications, besides it is a good indicator of prenatal quality. The objective of this study is knowing the frequency of CS notification at Antonio Pedro University Hospital of Federal Fluminense University (HUAP), Niterói, Rio de Janeiro, and analyse several data from the compulsory notification sheets (CNS) of this disease.

Methods Retrospective temporal study about the frequency of CS's notification in HUAP (Epidemiological Surveillance Department) in the period from 2008-2015.

Results We found 56 CNS. We analysed data on diagnosis, treatment, signs and symptoms of CS, among others. We exclude four sheets $(4 / 56 / 7.14 \%)$ because they do not contain minimum data for analysis. So, we worked with $52 \mathrm{CNS}$ of 
the eight-year period. Only $9(9 / 52 / 17.37 \%)$ CNS were fully met. The numbers of childbirth/CS/\% in HUAP were: 2008 (389/8/2.05\%); 2009 (373/6/1.60\%); $2010 \quad(442 / 4 / 0.90 \%)$; 2011 (508/0/0\%); 2012 (521/1/0.19\%); 2013 (640/9/1.40\%); 2014 (522/14/2.68\%); 2015 (422/10/2.37\%). Maternal age: 6 pregnant women (11.5\%) between 14 and 18 years, 25 (48.1\%) between 19 and 25 years, 18 (34.6\%) between 26 and 40 years and $3(5.8 \%)$ Ignored. About prenatal care: 10 pregnant women (19.2\%) performed in HUAP, 34 (65.4\%) in basic health units in Niterói and other cities in the state, and $8(15.4 \%)$ did not done. The diagnosis of maternal syphilis occurred during prenatal care in $37(71 \%)$ cases, in childbirth in $12(23 \%)$ and after childbirth in $3(6 \%)$. Only 11 partners (21.1\%) were treated. Forty-eight (92.3\%) newborns were treated appropriately. Cases evolutions: 46 (88.5\%) were still alive, $3(5.8 \%)$ were stillborn, $2(3.8 \%)$ evolved to postpartum death and $1(1.9 \%)$ was an abortion.

Conclusion The notification and complete padding of the CS's CNS is of crucial importance for CS control with the pregnant women and prenatal evaluation. We found many flaws for a federal university service.

\section{P3.115 PREVALENCE AND RISK FACTORS ASSOCIATED WITH BACTERIAL VAGINOSIS IN SOUTHERN MEXICO}

Karen Cortés Sarabia, Ana Karen Estrada Moreno, Miying Dessire Goméz Cervantes, Luz Del Carmen Alarcón Romero Eugenia Flores Alfaro, Amalia Vences Velázquez. Autonomous University of Guerrero, Chilpancingo, Mexico

\subsection{6/sextrans-2017-053264.350}

Introduction Bacterial vaginosis (BV) is the most common vaginal infection, characterised by a decrease in Lactobacilli and an increase in anaerobic bacteria mainly Gardnerella vaginalis, a variable Gram coccobacillus that is isolated in up to $98 \%$ of cases. The prevalence of this clinical entity is varied and the associated risk factors are: having multiple sexual partners, age, use of contraceptive methods, alcohol consumption, smoking habit, among others. The diagnosis of this clinical entity is important because of the multiple gyneco-obstetric complications associated with this clinical entity like abortion, infertility, ectopic pregnancies and the predisposition to acquire other sexually transmitted diseases such as herpes, HIV, HPV, and others.

Methods A total of 298 samples of vaginal swab from women between the ages of 16 to 65 years. The diagnosis of BV was performed using the Amsel and Nugent criteria, in which we evaluate the vaginal flow, $\mathrm{pH}$, amine test, the presence of clue cells and the quantification of bacterial morphotypes. The culture of Gardnerella vaginalis was performed in Columbia medium enriched with $10 \%$ of human blood and a specific supplement for the growth of this bacteria, for its identification we use oxidase, catalase and hippurate hydrolysis. The women who agreed to participate in the study signed an informed consent and the clinical and personal information was obtained through a survey. The results were analysed in the stata V.11 program.

Results We report a prevalence of BV of $10.4 \%$ in the population of Chilpancingo Guerrero in Mexico. The risk factors associated with this clinical entity were aged between 32 to 41 years (OR: $495 \% \mathrm{CI}=1.2-13.6 \mathrm{p}=0.025)$, having 2 to 3 sexual partners (OR: $3.495 \% \mathrm{CI} \quad 1.3-8.9 \quad \mathrm{P}=0.012)$ and smoking habit (OR: 3.4 95\% CI 1.1-10.6 p=0.039).
Conclusion The results obtained in this study provided information about the epidemiology of $\mathrm{BV}$, which contributed to the efective detection and treatment of this clinical entity.

\section{P3.116 IMPROVING ACCESS TO HIV DIAGNOSIS BY EXPANDING IMPLEMENTATION OF RAPID DIAGNOSTIC TESTS IN THE STATE OF SAO PAULO, BRAZIL (2006 TO 2016)}

Karina Wolffenbuttel, Márcia Fernandes dos Santos, Tânia Regina Corrêa de Souza, Ivone Aparecida de Paula, Maria Clara Gianna. STD/AIDS State Program, STD/AIDS Reference and Training Centre, São Paulo - SP, Brazil

\subsection{6/sextrans-2017-053264.351}

Issue Sao Paulo has 43 million inhabitants, 645 cities, 251.133 reported AIDS cases. Despite the HIV low prevalence in our state $(0.5 \%)$. The HIV rapid diagnostic testing method (RDT HIV) universal implementation is considered a very important strategy to increase access of vulnerable populations to HIV diagnosis together with other focused strategies. The goal was to implement the RDT HIV in at least one health public service in all 645 municipalities. In Brazil, we have the Health Unic System SUS as an universal public health system.

Description Between 2006-2016, around 500 trainers trained approximately 10.000 health professionals. From 2010 to 2016, the STD/AIDS Program Coordination trained 800 facilitators during the decentralisation process. In 2016586 municipalities implemented RDT HIV besides conventional testing (91\%); 60\% in primary care units. As proxy of HIV performance, we focus HIV annual campaigns carried out in the state since 2008 considering the majority adherence of municipalities (91\% in 2016) and health services. 7000 rapid diagnostic HIV tests in 2008;18 000 in 2009; 35000 in 2010; 40000 in 2011; 40000 in 2012; 156000 in 2013; 247000 in 2014; 251000 in 2015.

Lessons learned: Training of professionals through the training of trainers has boosted the implementation of HIV RDT. Monitoring RDT HIV implementation through five macroregional meetings was fundamental to find out and discuss local obstacles to offer access to HIV testing without having to schedule. Most vulnerable population for HIV continues to seek out ST/AIDS referral services for testing. Primary health care health workers have difficulty delivering positive HIV test results. Performing extra-mural actions is easier when the health unit has already implemented the test in its routine.

New steps Priorities for 2017: Continue the implementation process among all primary health care services. Expand and focus HIV testing In places of concentration and socialisation of more vulnerable groups such as gays and transvestites. Support outreach-testing activities by NGOs.

\section{P3.117 HIV TESTING CAMPAIGNS: AN IMPORTANT STRATEGY FOR THE EXPANSION OF EARLY DIAGNOSIS IN THE STATE OF SAO PAULO, BRAZIL - SIX CAMPAIGNS - TWO MILLION TESTES PERFORMED FROM 2008 TO 2016}

Karina Wolffenbuttel, Márcia Fernandes Dos Santos, Tânia Regina Corrêa De Souza, Maria Aparecida Da Silva Maria Clara Gianna. STD/AIDS State Program, STD/AIDS Reference and Training Centre, São Paulo - SP, Brazil

10.1136/sextrans-2017-053264.352 